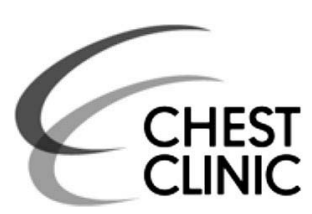

${ }^{1}$ Respiratory Medicine Unit (RCU), Hospital Umum Sarawak, Kuching, Malaysia ${ }^{2}$ Division of Paediatric Cardiology, Heart Center, Sarawak General Hospital, Kuching, Malaysia

\section{Correspondence to}

Dr Sze Shyang Kho, Respiratory Medicine Unit (RCU), Hospital Umum Sarawak, Kuching 93586, Malaysia; bzk99@hotmail.com

Received 8 December 2017 Revised 1 March 2018 Accepted 12 March 2018 Published Online First 24 March 2018

\title{
Pulmonary arteriovenous malformation presenting as spontaneous haemothorax on transthoracic ultrasound
}

\author{
Sze Shyang Kho, ${ }^{1}$ Mei Ching Yong, ${ }^{1}$ Swee Kim Chan, ${ }^{1}$ Martin Ngie Liong Wong, ${ }^{2}$ \\ Siew Teck Tie ${ }^{1}$
}

\section{CASE}

A 45-year-old man with no known chronic medical illness presented with insidious onset of exertional dyspnoea and left pleuritic chest pain. He was pale on physical examination and left chest percussion note was dull. Otherwise, he had no telangiectasia, cyanosis or finger clubbing. No family history of telangiectasia or bleeding episodes were reported, he also denied any history of epistaxis or haemoptysis. Chest roentograph on admission demonstrated left pleural effusion. Transthoracic ultrasound showed a large thin-walled elongated hypoechoic nodule surrounded by collapsed lung and loculated hyperechoic pleural fluid (figure 1A, arrow). Colour Doppler ultrasound established turbulent flow within the nodule (figure 1B, arrow) which raised the suspicion of pulmonary arteriovenous malformation (PAVM). His haemoglobin level was $5.5 \mathrm{~g} / \mathrm{dL}$. Urgent CT thorax revealed multiple PAVMs with the largest at the left lower lobe, arising from the left descending pulmonary artery (figure 1C, arrow). Diagnostic thoracocentesis confirmed haemothorax with pleural fluid to serum haematocrit ratio of 0.64 (figure 1D). The PAVM was confirmed during pulmonary angiographic study (figure 1E, arrow) and was successfully embolised (figure 1F). Medical thoracoscopy was performed subsequently for clot evacuation. The patient recovered and symptoms did not recur. The patient was advised for hereditary haemorrhagic telangiectasia (HHT) genetic testing.

PAVM is a rare clinical disease which manifests in various presentations from asymptomatic in mild cases, to cyanosis, congestive heart failure and

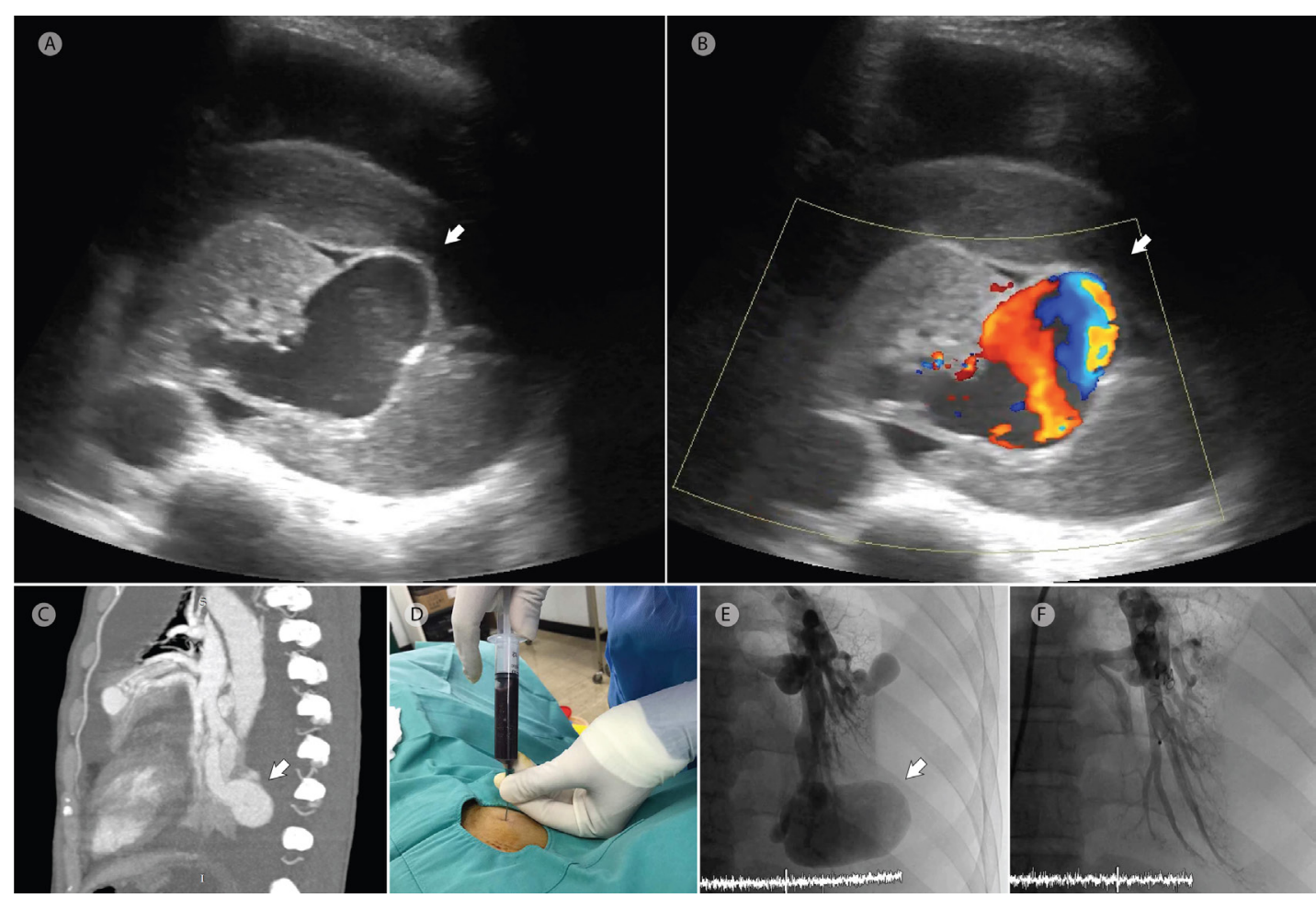

Check for updates

To cite: Kho SS, Yong MC, Chan SK, et al. Thorax 2018:73:892-893
Figure 1 Transthoracic ultrasound demonstrated an elongated hypoechoic nodule surrounded by collapsed lung and loculated hyperechoic pleural fluid ( $\mathbf{A}$, arrow) with turbulent flow during colour Doppler examination (B, arrow). CT Thorax revealed multiple pulmonary arteriovenous malformations (PAVM) with the largest at the left lower lobe, arising from the left descending pulmonary artery (C, arrow). Stale blood aspirated during thoracocentesis (D). The PAVM was visualised during pulmonary angiographic study (E, arrow) and was successfully embolised (F). 
respiratory failure in severe cases. ${ }^{1}$ Spontaneous haemothorax as a life-threatening complication of PAVM is even rarer. ${ }^{12}$ However, a population-wide cancer screening programme using low-dose thoracic CT estimated the prevalence of PAVM to be 1 per 2600 individuals, which is more common than previously thought. ${ }^{3}$ Hence, clinicians should always have high index of suspicion for PAVM when dealing with a spontaneous haemothorax.

CT thorax is generally considered the gold standard investigation for PAVM. ${ }^{4}$ However, colour Doppler ultrasound has recently been shown to be useful in a small series of patients in the diagnosis of PAVM. ${ }^{5}$ Besides, the mobility and point-of-care nature of ultrasound make it a useful bedside tool during initial assessment.

Approximately $70 \%$ of multiple PAVMs are associated with HHT. ${ }^{1}$ Although our patient did not fulfil clinical criteria for HHT, it is known that patients with HHT may remain subclinical at the time of presentation due to age-related penetrance. Hence, HHT cannot be reliably excluded on clinical grounds alone in patients presenting with multiple PAVMs alone without other clinical features suggesting of HHT. In such cases, HHT gene testing may be helpful. ${ }^{6}$ Moreover, although sporadic PAVM can occur in non-HHT patients, these are generally solitary. Common causes include: following cavopulmonary anastomosis for complex cyanotic heart disease in the lung without hepatic venous effluent, gestational trophoblastic disease, traumatic arteriovenous fistulae and other inherited vasculopathies. $^{46}$

Our case highlights the rare complication of a rare clinical condition; at the same time demonstrating the usefulness of transthoracic ultrasound in PAVM detection during initial assessment of a spontaneous haemothorax.

Contributors SSK initiated the idea for case reporting, prepared the final copy of the manuscript and was involved in the initial diagnosis of the subject. MCY and SKC finalised the final copy of the manuscript and was involved in the management of the patient. MNLW performed the pulmonary angiographic study. STT supervised the whole procedure and reviewed the final manuscript. All authors have read and approved the final manuscript.

Funding This research received no specific grant from any funding agency in the public, commercial or not-for-profit sectors.

Competing interests None declared.

Patient consent Obtained.

Provenance and peer review Not commissioned; externally peer reviewed.

(C) Article author(s) (or their employer(s) unless otherwise stated in the text of the article) 2018. All rights reserved. No commercial use is permitted unless otherwise expressly granted.

\section{REFERENCES}

1 Gossage JR, Kanj G, Ghassan K. Pulmonary arteriovenous malformations. A state of the art review. Am J Respir Crit Care Med 1998;158:643-61.

2 Khan AA, Hunt I, Hamdane K, et al. Massive pulmonary arteriovenous malformation presenting with tamponading haemothorax. Thorax 2007;62:836.

3 Nakayama M, Nawa T, Chonan T, et al. Prevalence of pulmonary arteriovenous malformations as estimated by low-dose thoracic CT screening. Int Med 2012:51:1677-81.

4 Shovlin CL. Pulmonary arteriovenous malformations. Am J Respir Crit Care Med 2014;190:1217-28.

5 Wang HC, Kuo PH, Liaw YS, et al. Diagnosis of pulmonary arteriovenous malformations by colour Doppler ultrasound and amplitude ultrasound angiography. Thorax 1998;53:372-6.

6 Shovlin CL, Condliffe R, Donaldson JW, et al. British thoracic society clinical statement on pulmonary arteriovenous malformations. Thorax 2017;72:1154-63. 